\title{
Decoherence in a single trapped ion due to engineered reservoir
}

\author{
X. X. Yi, D. L. Zhou, C. P. Sun \\ Institute of Theoretical Physics, Academia Sinica, P.O.Box 2735, Beijing 100080, China
}

\begin{abstract}
The decoherence in trapped ion induced by coupling the ion to the engineered reservoir is studied in this paper. The engineered reservoir is simulated by random variations in the trap frequency, and the trapped ion is treated as a two-level system driven by a far off-resonant plane wave laser field. The dependence of the decoherence rate on the amplitude of the superposition state is given.

PACS number(s):42.50.Vk, 03.65.-w,03.67.Lx
\end{abstract}

According to quantum mechanics[1], a system can exist in a superposition of distinct states, whereas these superposition state seems not to appear in the macroscopic world. One possible explanation of this para$\operatorname{dox}[2]$ is based on the fact that systems are never completely isolated but interact with the surrounding environment, which contains a large number of degrees of freedom. The environment influences the system evolution which continuously decoheres and transforms system superposition into statistical mixtures which behave classically $[2,3]$. There are many assumptions involved in modeling the coupling of the system to its environment. For example, the nature of the coupling of a system to its environment is generally taken to be a linear[4] or a nonlinear[5] function of the position operator of the object. Assumptions are also made about the environment. One example is to treat the environment as a reservoir of quantum oscillators, each of which interacts with the quantum system in question. Such a environment is extremely difficult to control because of the lack of knowledge about the environment and its coupling to a system. A recent work[6] extends the investigations of the decoherence beyond the ambient reservoirs and engineer the state of the reservoir, as well as the form of the system-reservoir coupling. In the work, they apply noisy potentials to the trap electrodes to simulate a hot reservoir, the system of trapped ion then feels a controllable reservoir, through which quantum superpositions are decohered into a state behaves classically.

In this letter, we present a theoretical study on decoherence of quantum superpositions of a single trapped ion through coupling to the engineered reservoir which is simulated by variating the trap frequency. We tackle the variations by treating them as a white noise, which results in decoherence of the trapped ion as you will see further . Before introducing a engineered reservoir, we consider a single ${ }^{9} \mathrm{Be}^{+}$ion confined in an $\mathrm{rf}($ paul) trap and interacting with a plane wave laser field. The Hamiltonian of this system may be written as (with $\hbar=1$ )

$$
\begin{aligned}
H & =\frac{p^{2}}{2 m}+V(r)+\frac{\omega_{e g}}{2}(|e\rangle\langle e|-| g\rangle\langle g|) \\
& +\frac{\Omega_{L}}{2} e^{i \omega_{L} t-i k_{L} r}|g\rangle\langle e|+H . c,
\end{aligned}
$$

where the first three terms describe the free motion of the ion with two levels $|e\rangle$ and $|g\rangle$ in a trap $V(r)=\frac{1}{2} m \omega^{2} r^{2}$, whereas the last two terms denote the coupling of the ion to the plane wave laser field. Based on this kind of models, nonclassical motional states such as thermal, Fock, coherent, squeezed and Schrödinger cat state are created $[7,8]$, it opens novel possibilities to study decoherence of quantum superposition[6]. For the plane wave excitation along the $\mathrm{x}$-axis, the motional effects along the $\mathrm{y}$ and $\mathrm{z}$ directions are unperturbed, in this case our Hamiltonian eq.(1) can simplifies to a one dimensional model

$$
\begin{aligned}
\tilde{H} & =\frac{p_{x}^{2}}{2 m}+V(x)+\frac{\delta}{2}(|e\rangle\langle e|-| g\rangle\langle g|) \\
& +\frac{\Omega_{L}}{2}(|e\rangle\langle g|+| g\rangle\langle e|)+p_{x} k_{L x}(|e\rangle\langle e|-| g\rangle\langle g|),
\end{aligned}
$$

where a unitary transformation

$$
U_{1}=e^{-\frac{i}{2}\left(\omega_{L} t-k_{L x} x\right)}|g\rangle\left\langle g\left|+e^{\frac{i}{2}\left(\omega_{L} t-k_{L x} x\right)}\right| e\right\rangle\langle e|
$$

has also been made. The detuning $\delta=\omega_{e g}-\omega_{L}$, the recoil shift $\frac{k_{L}^{2}}{2}$ is ignored here. The last term in eq.(2) results from Doppler effect, which leads to the coupling between the nearest neighbor motional states[9] and the decoherence of a single trapped atomic/ionic qubit[10]. In the following, we are concentrating on the situation where the detuning $\delta$ and the coupling strength $\Omega_{L}$ is large, especially, the coupling strength $\Omega_{L}$ is much larger than the coupling of the system to ambient reservoir, the latter condition is relevant to the recent engineered environment experiment for avoiding the ambient environment which leads to the natural decoherence[6]. Although the coupling length $(\sim K H z)$ is large, it is smaller than the trap frequency $(\sim \mathrm{MHz})$ so that the internal spin dynamics and the external motion of the ion in the trap occur on two different time scales. Therefore, it is useful to go to a rotating frame that eliminates the fourth term in eq.(2), which describes the Rabi oscillations between the two internal states. In the rotating frame, we will be able to understand more clearly how the last term in eq.(2), which couples the motional and the internal dynamics, leads to decoherence when the trap frequency $\omega$ is modulated to be random.

We go to the rotating frame by making a unitary transformation 


$$
U_{2}=e^{-i\left(\frac{\Omega_{L}}{2} \sigma_{x}+\frac{\delta}{2} \sigma_{z}\right) t},
$$

where $\sigma_{x}=|g\rangle\langle e|+| e\rangle\left\langle g\left|, \sigma_{z}=\right| e\right\rangle\langle e|-| g\rangle\langle g|$ are pauli matrices. In the rotating frame, the system evolves according to

$$
i \frac{\partial}{\partial t}\left|\psi^{I}(t)\right\rangle=H_{I}(t)\left|\psi^{I}(t)\right\rangle,
$$

where $H_{I}(t)$ is given by

$$
\begin{aligned}
H_{I}(t) & =\frac{p_{x}^{2}}{2 m}+V(x) \\
& +p_{x} k_{L x}\left(\alpha_{x}(t) \sigma_{x}+\alpha_{y}(t) \sigma_{y}+\alpha_{z}(t) \sigma_{z}\right) .
\end{aligned}
$$

The time varying coefficients $\alpha_{x}(t), \alpha_{y}(t), \alpha_{z}(t)$ are given by[11]

$$
\begin{gathered}
\alpha_{x}(t)=\frac{\Omega_{L} \delta}{\Omega_{e}^{2}}\left(1-\cos \left(\Omega_{e} t\right)\right), \\
\alpha_{y}(t)=\frac{\Omega_{l}}{\Omega_{e}} \sin \left(\Omega_{e} t\right), \\
\alpha_{z}(t)=\frac{\delta^{2}}{\Omega_{e}^{2}}+\frac{\Omega_{L}^{2}}{\Omega_{e}^{2}} \cos \left(\Omega_{e} t\right),
\end{gathered}
$$

where $\Omega_{e}=\sqrt{\Omega_{L}^{2}+\delta^{2}}$. In what follows, we make two simplifications in order to extract out the dominant behaviors of the system. Noticing the coefficients $\alpha_{i}(t), i=$ $x, y, z$ oscillate rapidly, we expect the system in the rotating frame to evolve on a much slower time scale than the period $2 \pi / \Omega_{e}$. In this sense we can simplify the Hamiltonian $H_{I}(t)$ given in eq.(6) by taking a average values of the coefficients $\alpha_{i}(t), i=x, y, z$, this is equivalent to coarse graining eq.(6). The coefficients $\alpha_{i}(t)$ then become time independent and reduced to $\alpha_{x}=\frac{\delta \Omega_{L}}{\Omega_{e}^{2}}, \alpha_{y}=0$, $\alpha_{z}=\frac{\delta^{2}}{\Omega_{e}^{2}}$. Furthermore, we make assumption that the system is being driven far-off-resonance, i.e, $\delta>>\Omega_{L}$. We therefore have

$$
H_{I}=\frac{p_{x}^{2}}{2 m}+V(x)+p_{x} k_{L x} \frac{\delta^{2}}{\Omega_{e}^{2}} \sigma_{z},
$$

we note $p_{x}=i \sqrt{\frac{m \omega}{2}}\left(a^{\dagger}-a\right)$ with $a\left(a^{\dagger}\right)$ the annihilation (creation) operator for motional state $|n\rangle$ which satisfies $\left(\frac{p_{x}^{2}}{2 m}+V(x)\right)|n\rangle=\omega\left(n+\frac{1}{2}\right)|n\rangle$, therefore the Hamiltonian(7) can be rewritten as

$$
H_{I}=\omega a^{\dagger} a+i g \sigma_{z}\left(a^{\dagger}-a\right),
$$

where $g=\sqrt{\frac{m \omega}{2}} \frac{\delta^{2}}{\Omega^{2}} k_{L x}$. $H_{I}$ couples nearest neighbors of motional state $|n\rangle$ with a same internal level $|g\rangle$ or $|e\rangle$. As will be clear further, the second term in $H_{I}$ leads to decoherence while the engineered reservoir applied. To show this, we first of all give the time evolution operator in the rotating frame,

$$
\begin{aligned}
U_{I}(t) & =e^{-i \omega a^{\dagger} a t}\left(|g\rangle\langle g| e^{f(t)} e^{A(t) a^{\dagger}} e^{B(t) a}\right. \\
& \left.+|e\rangle\langle e| e^{f(t)} e^{-A(t) a^{\dagger}} e^{-B(t) a}\right),
\end{aligned}
$$

where

$$
A(t)=\frac{i g}{\omega}\left(e^{i \omega t}-1\right), B(t)=-A^{*}(t),
$$

$$
f(t)=-i \frac{g^{2}}{\omega} t+\frac{g^{2}}{\omega^{2}}\left(1-e^{-i \omega t}\right) .
$$

We consider a initial state of the form in the rotating frame

$$
\left|\psi^{I}(0)\right\rangle=c_{g}|g\rangle \otimes\left|\alpha_{g}\right\rangle+c_{e}|e\rangle \otimes\left|\alpha_{e}\right\rangle,
$$

where $\left|\alpha_{i}\right\rangle,(i=g, e)$ denotes a coherent state, $c_{g}$ and $c_{e}$ are constants and satisfying $\left|c_{g}\right|^{2}+\left|c_{e}\right|^{2}=1$. This kind of states may be created by during Raman transitions [7]. Eqs(3),(4) and (9) together govern the evolution of the system. With these equations, we can analytically evolve the initial state (10) to obtain

$$
\begin{aligned}
|\psi(t)\rangle & =e^{\frac{i}{2} \omega_{L} t-i \omega a^{\dagger} a t}\left(\alpha_{1}(t) c_{g}(t)\left|\alpha_{g}^{-}(t)\right\rangle\right. \\
& \left.+\alpha_{2}(t) c_{e}(t)\left|\alpha_{e}^{-}(t)\right\rangle\right) \otimes|g\rangle \\
& +e^{-\frac{i}{2} \omega_{L} t-i \omega a^{\dagger} a t}\left(\alpha_{2}(t) c_{g}(t)\left|\alpha_{g}^{+}(t)\right\rangle\right. \\
& \left.+\alpha_{1}^{*}(t) c_{e}(t)\left|\alpha_{e}^{+}(t)\right\rangle\right) \otimes|e\rangle,
\end{aligned}
$$

where

$$
\begin{gathered}
\alpha_{1}(t)=\cos \frac{\Omega_{e}}{2} t-\frac{i \delta}{\Omega_{e}} \sin \frac{\Omega_{e}}{2} t, \alpha_{2}(t)=-i \frac{\Omega_{L}}{\Omega_{e}} \sin \frac{\Omega_{e}}{2} t \\
c_{g}(t)=c_{g} e^{f(t)-\frac{1}{2}|A(t)|^{2}}, \\
c_{e}(t)=c_{e} e^{f(t)-\frac{1}{2}|A(t)|^{2}}, \\
\alpha_{g}^{ \pm}(t)=\alpha_{g}+A(t) \pm \frac{i}{2} k_{L x} \sqrt{\frac{1}{2 m \omega}} e^{-i \omega t} \\
\alpha_{e}^{ \pm}(t)=\alpha_{e}-A(t) \pm \frac{i}{2} k_{L x} \sqrt{\frac{1}{2 m \omega}} e^{-i \omega t} .
\end{gathered}
$$

Eq.(11) is the main result of our study, with which we can explain the essential properties of the system. As eq.(11) shows, the initial motional state $\left|\alpha_{g}\right\rangle$ and $\left|\alpha_{e}\right\rangle$ are displaced to be $\left|\alpha_{g}^{ \pm}(t)\right\rangle$ and $\left|\alpha_{e}^{ \pm}(t)\right\rangle$, respectively. Their displacements depend on the internal state of trapped ion. We are now interested in the coefficient of the off-diagonal element of the density operator $\rho(t)=|\psi(t)\rangle\langle\psi(t)|$ in ionic internal space, its module which represents qualitatively decoherence of the system is 


$$
\begin{aligned}
R(\omega, t) & =\operatorname{Mod}\left\{\alpha_{1}^{*}(t) \alpha_{2}(t)\left|c_{g}\right|^{2}\left\langle\alpha_{g}^{-}(t) \mid \alpha_{g}^{+}(t)\right\rangle e^{-i \omega \alpha_{g}^{+}(t) \alpha_{g}^{-*}(t) t}\right. \\
& +\alpha_{1}^{* 2}(t) c_{g}^{*} c_{e}\left\langle\alpha_{g}^{-}(t) \mid \alpha_{e}^{+}(t)\right\rangle e^{-i \omega \alpha_{e}^{+}(t) \alpha_{g}^{-*}(t) t} \\
& +\left|\alpha_{2}(t)\right|^{2} c_{e}^{*} c_{g}\left\langle\alpha_{e}^{-}(t) \mid \alpha_{g}^{+}(t)\right\rangle e^{-i \omega \alpha_{e}^{-}(t) \alpha_{g}^{+*}(t) t} \\
& +\alpha_{1}^{*}(t) \alpha_{2}^{*}(t)\left|c_{e}\right|^{2}\left\langle\alpha_{e}^{-}(t) \mid \alpha_{e}^{+}(t)\right\rangle \\
& \left.\cdot e^{-i \omega \alpha_{e}^{-}(t) \alpha_{e}^{+*}(t) t}\right\} e^{-4 \frac{g^{2}}{\omega^{2}}(1-\cos \omega t)}
\end{aligned}
$$

Where $\operatorname{Mod}\{\ldots\}$ denotes the module of the term in the brace. We take a special case $\alpha_{g}=\alpha_{e}=2$ with fixed $\omega=2 \pi \times 11.3 \mathrm{MHz}$ as an example to illustrate $R(\omega, t)$ vs. time in figure 1 . As figure 1 shows, $R(\omega, t)$ is a periodic function of time $t$, which period depends on the parameters chosen. During the first few Rabi cycles, the coherent state parameters $\alpha_{g}^{ \pm}(t) \simeq \alpha_{g} \alpha_{e}^{ \pm}(t) \simeq \alpha_{e}$, so that the internal and external degrees of freedom appear to be decoupled and the system simply oscillates rapidly between internal states, this was shown in the first envelope of figure 1. However, for a long time scales, the coupling between the internal and external states make effects, this result in a modulation of the Rabi oscillations. Up to now, the engineered reservoir does not take place and the results illustrated in figure 1 indicate that without engineered reservoir there are not any decoherences occur in the system.

A engineered reservoir coupled to the trapped ion is simulated by variations in the trap frequency, oscillating near the ion's original trapped frequency. Physically, decoherence in this case arises from random perturbations of the Hamiltonian. In what follows, we want to model the effects of the variations in the trap frequency with the same formalism in ref.[12]. Our main idea is to treat the variations in the trap frequency as fluctuations. For an ion in a Paul trap[6], Colorado group realizes the variations by a random voltage noise source applied to the trap electrodes, the noise source is passed through a lowpass filter network with a cut-off frequency well below the trap frequency. The atom then sees a harmonic potential with fluctuating spring constant. The Hamiltonian for a trapped ion in a harmonic potential with fluctuating spring constant in the rotating frame is

$$
H_{I}=\frac{p_{x}^{2}}{2 m}+V(x)+p_{x} k_{L x} \frac{\delta^{2}}{\Omega_{e}^{2}} \sigma_{z}+\frac{1}{2} m \omega^{2} \varepsilon(t) x^{2},
$$

this Hamiltonian is just the eq.(7) plus a term which describes the fluctuations in the trap frequency. If we take the fluctuations as a white noise, i.e.,

$$
\varepsilon(t) d t=\sqrt{\Gamma} d W(t)
$$

and set

$$
X=\sqrt{\frac{m \omega}{2}} x, \quad P_{x}=(2 m \omega)^{-\frac{1}{2}} p_{x},
$$

the Hamiltonian (14) becomes

$$
\begin{aligned}
H & =\omega\left(P_{x}^{2}+X^{2}\right)+g P_{x} \sigma_{z}+\sqrt{\Gamma} \omega X^{2} d W(t) \\
& =H_{0}+\sqrt{\Gamma} \omega X^{2} d W(t) .
\end{aligned}
$$

Here, $d W(t)$ is the increment of a real Wiener process[13], $g=\sqrt{2 m \omega} k_{L x} \frac{\delta^{2}}{\Omega_{e}^{2}}$ and $\Gamma$ scales the fluctuations. For a single run with a known behavior of the fluctuations in time, we use a stochastic Schrödinger equation in the Ito formalism[14]

$d \rho_{I}(t)=-\frac{i}{\hbar}\left[H_{0}, \rho_{I}\right]-\frac{i \sqrt{\Gamma}}{\hbar}\left[X^{2}, \rho_{I}\right] d W(t)-\frac{\Gamma}{2}\left[X^{2}\left[X^{2}, \rho_{I}\right]\right]$

to describe the time evolution of the density operator $\rho_{I}$ in the rotating frame. Here, we are not interested in the effects of the fluctuation in short time scale, in this sense, we may take a average over the fluctuation to get the master equation for the average density operator $\rho^{a}$

$$
\frac{d \rho^{a}}{d t}=-i\left[H_{0}, \rho^{a}\right]-\Gamma\left[X^{2}\left[X^{2}, \rho^{a}\right]\right] .
$$

We want to determine the off-diagonal element of the density operator $\rho=U_{2} \rho^{a} U_{2}^{\dagger}$ in the internal space spanned by $|e\rangle$ and $|g\rangle$, where $U_{2}$ is given by eq.(4). To do this, we first derive a system of equations for $\rho_{i j}^{a}=\left\langle i\left|\rho^{a}\right| j\right\rangle,(i, j=g, e)$

$$
\begin{aligned}
& \frac{d \rho_{g e}^{a}}{d t}=2 i g P_{x} \rho_{g e}-\frac{\Gamma^{2}}{2} \omega^{2}\left[X^{2}\left[X^{2}, \rho_{g e}^{a}\right]\right. \\
& \frac{d \rho_{e g}^{a}}{d t}=-2 i g P_{x} \rho_{e g}-\frac{\Gamma^{2}}{2} \omega^{2}\left[X^{2}\left[X^{2}, \rho_{e g}^{a}\right]\right. \\
& \frac{d \rho_{i i}^{a}}{d t}=-\frac{\Gamma^{2}}{2} \omega^{2}\left[X^{2}\left[X^{2}, \rho_{i i}^{a}\right],(i=e, g)\right.
\end{aligned}
$$

It is easy to show that the off-diagonal element of the density operator $\rho$ can be represented as

$$
\begin{aligned}
\langle g|\rho| e\rangle & =\alpha_{1}^{*}(t) \alpha_{2}^{*}(t) \rho_{g g}^{a}(t)+\left[\alpha_{1}^{*}(t)\right]^{2} \rho_{g e}^{a}(t) \\
& +\left|\alpha_{2}(t)\right|^{2} \rho_{e g}^{a}(t)+\alpha_{1}^{*}(t) \alpha_{2}(t) \rho_{e e}^{a}(t) .
\end{aligned}
$$

For a short time scale, $\left\langle n\left|\rho_{i j}^{a}(t)\right| m\right\rangle \sim 0$, for $m \neq n$, The module $R(t)$ of the off-diagonal element which characterizes the decoherence is given by

$$
\begin{aligned}
R(t) & =\operatorname{Mod}\left\{\operatorname{Tr}_{e}\langle g|\rho| e\rangle\right\} \\
& =\operatorname{Mod}\left\{\sum _ { n = 0 } ^ { \infty } \left[\alpha_{1}^{*}(t) \alpha_{2}^{*}(t)\left\langle n\left|\rho_{g g}^{a}(0)\right| n\right\rangle\right.\right. \\
& +\left[\alpha_{1}^{*}(t)\right]^{2}\left\langle n\left|\rho_{g e}^{a}(0)\right| n\right\rangle \\
& +\left|\alpha_{2}(t)\right|^{2}\left\langle n\left|\rho_{e g}^{a}(0)\right| n\right\rangle \\
& \left.\left.+\alpha_{1}^{*}(t) \alpha_{2}(t)\left\langle n\left|\rho_{e e}^{a}(0)\right| n\right\rangle\right] e^{-\Gamma \omega^{2}\left(n^{2}+n+1\right) t}\right\},
\end{aligned}
$$

where $\alpha_{1}(t), \alpha_{2}(t)$ and $|n\rangle$ are the same as above mentioned, $\operatorname{Tr}_{e}$ denotes a trace over the external states. As 
eq.(21) shows, the decoherence rate $\Gamma \omega^{2}\left(n^{2}+n+1\right)$ depends on the character of the fluctuations, the trap frequency and the motional state of the trapped ion. Physically, the trap frequency play a role of the coupling of the system to the reservoir, so the larger the trap frequency, the larger the decoherence rate(decay rate). The fact that the decoherence rate depends on the motional state of trapped ion was observed in the experiments of ref.[7]. Again, we consider the state given by eq.(10) as a initial state, the numerical results of eq.(20) are illustrated in Fig.2, there are a few Rabi cycles in the beginning. However, for a long time scales, the oscillations disappear, it is evidence that decoherence occur in the system.

To sum up, decoherence in a two-level trapped ion is studied in this paper. The decoherence is induced by coupling the ion to the engineered reservoir, which is simulated by random variations in the trap frequencies. Without this reservoir, the transitions between the ionic internal levels manifest modulated Rabi transition, whereas the transition was suppressed when the engineered reservoir take place. The suppressed transitions indicate that there is decoherence in the trapped ion system.

ACKNOWLEDGEMENT: This work was supported by Chinese postdoctoral Fund via Institute of Theoretical Physics, Academia Sinica. Discussions with Prof. W.M.Zheng, Dr. S. X. Yu and Dr. Y.X. Liu are gratefully acknowledged.

[1] L.D.Landau and E.M.Lifshitz, Quantum Mechanics (Pergamon press,1977) Third revised edition.

[2] W.H.Zurek, Phys. Today 44, No.10 (1991)36.

W.H.Zurek, Phys. Rev. D 24 (1981) 1516, ibid26 (1982) 1862.

C.P.Sun, X.X.Yi and X.J.Liu, Fortschr. Phys. 43 (1995) 585.
[3] D.F.Walls, G.J.Milburn, Phys. Rev. A 31(1985) 2403. A.O.Caldeira, A.J.Leggett, Phys. Rev. A 31(1985)1509.

[4] A.O.Calderia, A.J.Leggett, Physica A 121(1983)587. E.Joos, H.D.Zeh, Z. Phys. B 59(1985)223. W.G.Unruh, W.H.Zurek, Phys. Rev. D 40(1989)1071. J.P.Paz, S.Habib, W.H.Zurek, Phys. Rev. D 47(1993) 488.

[5] B. L. Hu, J. P. Paz, Y. H. Zhang, Phys. Rev. D 47 (1993) 1576.

L.M.Kuang, H.S.Zeng, Z.Y.Tong, Phys. Rev. A 60(1999)3815.

[6] C.J.Myatt, B.E.King, Q.A.Turchette, C.A.Sackett, D.Kielpinski, W.M.Itano, C.Monroe, D.J.Wineland, Nature 403(2000)1269.

[7] D. M. Meekhof, C. Monroe, B. E. King, W. M. Itano, D. J. Wineland, Phys. Rev. Lett. 76(1996)1796. C.Monroe, D.M.Meekhof, B.E.King, D.J.Wineland, Science 272(1996)1131.

[8] R.L.de Matos Filho, W.Vogel Phys. Rev. Lett. $76(1996) 608$.

[9] J.Javanainen etal., J.Opt.Soc. Am. B 1(1984)111.

[10] L. You, quant-ph/0001117.

[11] J.Williams, R.Walser, J.Cooper, E.A.Cornell, M.Holland, Phys. Rev. A 61(2000)033612.

[12] S.Schneider, G.J.Milburn, Phys. Rev. A 57 (1998) 3748. ibid, 59(1999) 3766.

[13] C.W. Gardiner, Handbook of stochastic process for physics, Chemistry and the natural science (SpringerVerlag, Berlin,1985).

[14] S.Dyrting, G.J.Milburn, Quantum and Semiclass. Opt. $8(1996) 541$.

\section{Figure captions:}

Fig.1: The module of the off-diagonal element of the density operator is plotted as a function of time with fixed trap frequency. The parameters chosen are $\alpha_{g}=\alpha_{e}=3$, $\omega=2 \pi \times 11.3 \mathrm{MHz}, \delta=4.0 \mathrm{GHz}, \Omega_{L}=10.0 \mathrm{KHz}$.

Fig.2: This plot shows the decoherence in trapped ion induced by the engineered reservoir. The module of the off-diagonal element of the density operator is calculated by the master equation. This plot shows the module as a function of time. The parameters chosen are the same as in figure 1 and $\Gamma=1.0 \mathrm{KHz}$. 